\section{Should All High-Risk Patients Receive Acetylsalicylic Acid 81 mg Daily for Primary Prevention of Cardiovascular Disease?}

\section{THE “PRO" SIDE}

A cornerstone for the discussion of using acetylsalicylic acid (ASA) for primary prevention of cardiovascular disease is determining which patients are at high risk and then determining if low-dose ASA would reduce that risk. Stratification of cardiovascular disease risk is a strategy employed by many health care providers to determine if individual patients are at risk of experiencing a cardiovascular event, given known risk factors such as age, smoking, hypertension, dyslipidemia, and diabetes mellitus. Many risk stratification tools are available, the most commonly used in Canada being the Framingham cardiovascular disease risk estimation tool. This tool (www.framinghamheartstudy. $\mathrm{org} / \mathrm{risk} /$ index.html), as well as many other risk calculators for various cardiovascular outcomes of interest, was developed by the Framingham Heart Study investigators. A high-risk population is generally defined as having a 10 -year risk of cardiovascular disease of $20 \%$ or greater, with myocardial infarction being the most prevalent event within the spectrum of cardiovascular disease that would lead to high-risk status.'

Low-dose ASA (defined as less than $325 \mathrm{mg} /$ day) has been shown in a meta-analysis to decrease the composite risk of myocardial infarction, stroke, or death from a vascular cause in both men and women when used for primary prevention. ${ }^{2}$ The authors of the meta-analysis reviewed the risks and benefits of ASA in various subgroups, including the baseline risk of coronary artery disease. Unfortunately, only $2 \%$ of patients in all of the studies included in the meta-analysis were in the high-risk category as defined above. There were no statistically significant differences between this group and patients receiving placebo in rates of serious vascular events, but the authors concluded that the statistical reliability of their analysis was compromised by the small sample size. ${ }^{2}$ It is therefore difficult to use data from meta-analyses to answer the question posed in this "Point Counterpoint" debate. Instead, the following argument is based on results from individual studies examining patient populations with various cardiovascular risk factors.

The Primary Prevention Project was one trial that examined the use of ASA $100 \mathrm{mg}$ daily in a population with risk factors for coronary artery disease and no history of cardiovascular disease. ${ }^{3}$ Although the report did not specify how many of the patients were at high risk, the trial included 4495 participants aged 45-94 years, all of whom had at least 1 risk factor for coronary artery disease (with 39\% of participants having 2 risk factors and $30 \%$ having 3 or more). The following risk factors were present: age at least 65 years (50\%), hypertension (69\%), smoker (15\%), dyslipidemia (41\%), diabetes (17\%), and family history of premature myocardial infarction $(11 \%){ }^{3}$ Two other trials enrolled higher-risk patients, but also included a small number of patients with coronary artery disease, which made it difficult to assess the data in terms of primary prevention. ${ }^{45}$

The Primary Prevention Project was stopped early (after 3.6 years) for ethical reasons, ${ }^{3}$ on the basis of newly published data demonstrating the benefit of ASA for primary prevention in individuals with cardiovascular risk factors. ${ }^{4,5}$ The Primary Prevention Project did not meet its primary end point of reducing the composite of cardiovascular death, nonfatal myocardial infarction, and nonfatal stroke (odds ratio [OR] 0.71, 95\% confidence interval [CI] 0.48-1.04). Importantly, however, the predefined secondary end point of cardiovascular death was reduced (OR 0.56, 95\% CI 0.31-0.99, $p=0.049$ ), as was the composite end point of total cardiovascular events and diseases (encompassing the primary outcome plus angina, transient ischemic attack, peripheral arterial disease, and revascularization procedure) (OR 0.77, CI 95\% 0.62-0.95, $p=0.014$ ). In the Primary Prevention Project, the most clinically important outcome, death, was reduced in a high-risk population with the daily use of low-dose ASA. ${ }^{3}$

One risk of using low-dose ASA is major bleeding. The risk of upper gastrointestinal bleeding in the general population is about 1 in 1000 or $0.1 \%$ per year. ${ }^{6}$ On the basis of population data and clinical trials, this risk is known to increase 2- to 3-fold with daily use of low-dose ASA. However, this increase in risk is low compared with the event rates of cardiovascular death and total cardiovascular events in a high-risk population. In the Primary Prevention Project, major nonfatal bleeding occurred in $1.1 \%$ of ASA users but only $0.3 \%$ of those assigned to receive placebo ( $p=0.0008$, number needed to harm 125$)$, much lower than the total rate of cardiovascular events $(6.3 \%$ among ASA users and $8.2 \%$ among placebo users). ${ }^{3}$ If a patient has additional risk factors for bleeding, the risk of bleeding may increase to a much higher and unacceptable rate if low-dose daily ASA is added. Therefore, although ASA is beneficial in a high-risk patient population, it is important to assess each patient's risk of bleeding (based on use of nonsteroidal antiinflammatory drugs, history of peptic ulcer disease, and use of warfarin or corticosteroids) before recommending low-dose ASA, to ensure that the bleeding risk does not outweigh any potential benefit of ASA.

Other cardiovascular therapies, such as statins, have taken a more prominent role in the primary prevention of cardiovascular disease, but they were not routinely used when the ASA trials 
described above were conducted. Accordingly, it is difficult to quantify the benefit of low-dose ASA in a high-risk patient today. ${ }^{2}$ Fortunately, ASA remains an affordable option for highrisk patients, costing about 10 cents per day. New trials are under way to further quantify the effect of ASA in primary prevention in 2 specific populations. The Aspirin to Reduce Risk of Initial Vascular Events (ARRIVE) trial is a large ongoing clinical trial to evaluate the use of low-dose ASA (100 mg daily) in the primary prevention of cardiovascular events in 12000 patients at moderate risk (defined as $10 \%-19 \%$ over 10 years), but without a history of cardiovascular disease. ${ }^{7}$ The ASPirin in Reducing Events in the Elderly (ASPREE) trial is evaluating the use of low-dose ASA (100 mg daily) in 19000 healthy patients over age 70 for overall benefit versus risk. ${ }^{8}$

As pharmacists, we need to make recommendations to patients and then allow them to ultimately decide if using daily low-dose ASA is right for them. ASA $81 \mathrm{mg}$ is the most commonly available low-dose formulation available on the Canadian market and is indicated by Health Canada for reducing the risk of a first nonfatal myocardial infarction in individuals deemed to be at high risk of such an event. Previous studies have shown that high-risk patients (those with hypertension, dyslipidemia, diabetes, and history of smoking) are at the highest risk of cardiovascular disease. ${ }^{1}$ Risk stratification is required to identify these patients, and conducting a bleeding assessment is also important when considering low-dose ASA therapy. The Canadian Cardiovascular Society endorses the use of ASA "in special circumstances in men and women without evidence of manifest vascular disease in whom vascular risk is considered high and bleeding risk is considered low."' This approach is appropriate, given the available evidence demonstrating reductions in both cardiovascular events and deaths with use of ASA. ${ }^{3}$

In conclusion, does an Aspirin a day keep the doctor away in the high-risk patient who requires primary prevention? The answer is yes, and this approach is appropriate for patients who are at low risk of bleeding.

\section{References}

1. Yusef S, Hawken S, Ounpuu S, Dans T, Avezum A, Lanas F, et al.; INTERHEART Study Investigators. Effect of potentially modifiable risk factors associated with myocardial infarction in 52 countries (the INTERHEART study): case-control study. Lancet 2004;364(9438):937-952.

2. Antithrombotic Trialists' (ATT) Collaboration. Aspirin in the primary and secondary prevention of vascular disease: collaborative meta-analysis of individual participant data from randomised trials. Lancet 2009; 373(9678):1849-1860.

3. de Gaetano G, Collaborative Group of the Primary Prevention Project. Lowdose aspirin and vitamin $\mathrm{E}$ in people at cardiovascular risk: a randomised trial in general practice. Lancet 2001;357(9250):89-95. Erratum in: Lancet 2001;357(9262):1134.

4. Hansson L, Zanchetti A, Carruthers SG, Dahlöf B, Elmfeldt D, Julius S, et al.; HOT Study Group. Effects of intensive blood-pressure lowering and lowdose aspirin in patients with hypertension: principal results of the Hypertension Optimal Treatment (HOT) randomised trial. Lancet 1998:351(9118):1755-1762.

5. Medical Research Council's General Practice Research Framework. Thrombosis prevention trial: randomised trial of low-intensity oral anticoagulation with warfarin and low-dose aspirin in the primary prevention of ischaemic heart disease in men at increased risk. Lancet 1998;351(9098):233-241.

6. Hernández-Díaz S, García Rodríguez LA. Cardioprotective aspirin users and their excess risk of upper gastrointestinal complications. BMC Med 2006;4:22.

7. ARRIVE: Aspirin to Reduce Risk of Initial Vascular Events [study website]. Bayer HealthCare; [cited 2011 Dec 6]. Available from: www.arrivestudy.com/EN/study.cfm

8. ASPirin in Reducing Events in the Elderly: ASPREE [study website]. Melbourne (Australia): Monash University, Department of Epidemiology and Preventive Medicine; [cited 2011 Dec 6]. Available from: www.aspree.org/AUS/aspree-content/aspree-study-details/about-aspree.aspx

9. Bell AD, Roussin A, Cartier R, Chan WS, Douketis JD, Gupta A, et al. The use of antiplatelet therapy in the outpatient setting: Canadian Cardiovascular Society guidelines. Can J Cardiol 2011;27 Suppl A:S1-S59.

Ann Thompson, BScPharm, PharmD, ACPR

Clinical Assistant Professor

Faculty of Pharmacy and Pharmaceutical Sciences

University of Alberta

Edmonton, Alberta

\section{THE "CON" SIDE}

Recent literature has called into question the utility of acetylsalicylic acid (ASA) in primary prevention of cardiovascular disease. ${ }^{1}$ These data have highlighted the need for a re-evaluation of the risk-benefit ratio of this therapeutic strategy. ${ }^{1}$ Given the current body of evidence, there is little evidence to support the use of low-dose ASA for primary prevention in any patient population, including patients considered to be at high risk for cardiovascular disease.

To date, 6 studies evaluating the use of ASA in primary prevention have been published..$^{2-7}$ These studies recruited patients between 1978 and 1995 and were published between 1988 and 2005. Although each of the studies examined the role of ASA in primary prevention, they were otherwise heterogeneous, involving different patient populations, using various ASA doses, and evaluating different clinical outcomes. The majority $(82 \%)$ of all patients in these trials were at low risk, defined as an annual rate of cardiovascular events less than $1 \%$. Only $4 \%$ were deemed to be at high risk (i.e., cardiovascular event rate greater than $2 \%$ per year ${ }^{1}$ ). In addition, only half of the trials included both men and women as study participants. The ASA dose used in these trials ranged from $100 \mathrm{mg}$ every other day to $500 \mathrm{mg}$ daily. Two of the studies used a $75-\mathrm{mg}$ dose, but no study used the commonly prescribed 81-mg dose. Careful examination of study outcomes reveals that 5 of the 6 studies had no statistically significant difference in predefined primary outcome between patients receiving ASA and those receiving placebo or other therapy. Additionally, any benefit that was seen was not clearly consistent across the studies. Interestingly, this lack of difference in outcomes is one of the reasons cited by the US Food and Drug Administration when it denied Bayer's indication application for ASA in primary prevention. ${ }^{8}$ Thus, although Canada has an indication for ASA in primary prevention, the United States still does not. Even if secondary outcomes 
were used to guide decision-making, the magnitude of benefit would be very small. For example, the numbers need to treat (NNT) to prevent certain cardiovascular outcomes over 1 year would be substantial: NNT $=2000$ to prevent one nonfatal myocardial infarction, NNT $=1666$ to prevent any major coronary event, and NNT $=1428$ to prevent any serious vascular event. ${ }^{1}$

From a public health perspective, one might argue that these NNT values, though relatively large, are acceptable, given that cardiovascular disease is one of the main drivers of morbidity and mortality in Canada. However, we must also consider the risks associated with using ASA in primary prevention of cardiovascular disease. These same studies indicate that the risk of major bleeding, either gastrointestinal or extracranial, potentially outweighs any benefit from a decrease in cardiovascular events. ${ }^{2-7}$ More specifically, the number needed to harm (NNH) in 1 year is 3333 for gastrointestinal or extracranial bleeding and 10000 for hemorrhagic stroke. ${ }^{1}$ It must also be kept in mind that these numbers are likely underestimations of bleeding events, given that the populations studied in these trials were relatively young and healthy. ${ }^{2-7}$ In fact, data suggest that risk of bleeding increases exponentially for every decade of age from 50 years on. ${ }^{9}$ The presence of gastrointestinal pain and uncomplicated or complicated ulcers, as well as the use of nonsteroidal anti-inflammatory drugs (NSAIDs), further increases the risk of gastrointestinal bleeding 2- to 10 -fold. ${ }^{9}$ Many clinicians place a higher value on preventing a cardiovascular event than on avoiding gastrointestinal bleeding, given the "reversibility" of the latter condition. However, this type of bleeding accounts for significant morbidity and mortality. Population studies of hospital admissions have shown that NSAIDS and ASA account for 121.9 major gastrointestinal events per 100000 person-years, resulting in death in $5 \%$ of cases. ${ }^{10}$ Furthermore, of these deaths, one-third can be attributed to use of low-dose ASA. This is especially important to keep in mind when ASA is being used for primary prevention, given that such therapy represents treatment of an otherwise "healthy" individual and is thus contrary to the Hippocratic principle of "first, do no harm".

Even if ASA truly decreases cardiovascular outcomes, even to a small extent, this difference would likely be overshadowed by contemporary treatment, including the use of statins and angiotensin-converting enzyme inhibitors, which were not widely available or used when the ASA trials were conducted. Unfortunately, the risk of bleeding is unlikely to have changed much over this period. The end result is an overestimation of benefit and an underestimation of bleeding risk with ASA for primary prevention. Recent guidelines from the Canadian Cardiovascular Society attempt to help clinicians in making decisions about ASA therapy for primary prevention. ${ }^{11}$ For example, a Class III, Level A recommendation states that for men and women without evidence of manifest vascular disease, "ASA at any dose is not recommended for routine use to prevent ischemic vascular events". The guidelines also suggest that there is Class IIb, Level C (consensus) evidence to consider, in special circumstances, low-dose ASA for those at high cardiovascular risk and low bleeding risk. Either way, both of these recommendations, as well as the existing scientific literature, argue against providing ASA $81 \mathrm{mg}$ daily to all high-risk patients for primary prevention of cardiovascular disease.

\section{References}

1. Antithrombotic Trialists' (ATT) Collaboration. Aspirin in the primary and secondary prevention of vascular disease: collaborative meta-analysis of individual participant data from randomised trials. Lancet 2009; 373(9678):1849-1860.

2. Peto R, Gray R, Collins R, et al. Randomised trial of prophylactic daily aspirin in British male doctors. Br Med J (Clin Res Ed) 1988;296(6618): 313-316.

3. Steering Committee of the Physicians' Health Study Research Group. Final report on the aspirin component of the ongoing Physicians' Health Study. N Engl J Med 1989;321(3):129-135.

4. Medical Research Council's General Practice Research Framework. Thrombosis prevention trial: randomised trial of low-intensity oral anticoagulation with warfarin and low-dose aspirin in the primary prevention of ischaemic heart disease in men at increased risk. Lancet 1998;351(9098):233-241.

5. Hansson L, Zanchetti A, Carruthers SG, Dahlöf B, Elmfeldt D, Julius S, et al.; HOT Study Group. Effects of intensive blood-pressure lowering and low-dose aspirin in patients with hypertension: principal results of the Hypertension Optimal Treatment (HOT) randomised trial. Lancet 1998;351(9118):1755-1762.

6. de Gaetano G, Collaborative Group of the Primary Prevention Project. Low-dose aspirin and vitamin $\mathrm{E}$ in people at cardiovascular risk: a randomised trial in general practice. Lancet 2001;357(9250):89-95. Erratum in: Lancet 2001;357(9262):1134.

7. Ridker PM, Cook NR, Lee IM, Gordon D, Gaziano JM, Manson JE, et al. A randomized trial of low-dose aspirin in the primary prevention of cardiovascular disease in women. $N$ Engl J Med 2005;352(13):1293-1304.

8. Cardiovascular and Renal Drugs Advisory Committee meeting. Bethesda (MD): US Food and Drug Administration; 2003 Dec 8 [cited 2011 Dec 15] Available from: www.fda.gov/ohrms/dockets/ac/03/questions/ 4012Q1_Final.htm

9. Hernández-Díaz S, García Rodríguez LA. Cardioprotective aspirin users and their excess risk of upper gastrointestinal complications. BMC Med 2006 [cited 2011 Dec 15];4:22. Available from: www.biomedcentral.com/ $1741-7015 / 4 / 22$

10. Lanas A, Perez-Aisa MA, Feu F, Ponce J, Saperas E, Santolaria S, et al.; Investigators of the Asociación Española de Gastroenterología (AEG). A nationwide study of mortality associated with hospital admission due to severe gastrointestinal events and those associated with nonsteroidal antiinflammatory drug use. Am J Gastroenterol 2005;100(8):1685-1693.

11. Bell AD, Roussin A, Cartier R, Chan WS, Douketis JD, Gupta A, et al. The use of antiplatelet therapy in the outpatient setting: Canadian Cardiovascular Society guidelines. Can J Cardiol 2011;27 Suppl A:S1-S59.

Sheri Koshman, BScPharm, PharmD, ACPR

Division of Cardiology

Faculty of Medicine and Dentistry

University of Alberta

Edmonton, Alberta 